\title{
Ponto de Paz: descrição prática de uma nova técnica
}

\author{
Paz's suture: practical description of a new technique \\ Punto de Paz: descripcíon prática de una nueva técnica
}

Recebido: 19/08/2021 | Revisado: 24/08/2021 | Aceito: 27/08/2021 | Publicado: 29/08/2021

Pedro Jordão de Almeida Silva Paz

ORCID: https://orcid.org/0000-0003-0959-4821

Centro Universitário Maurício de Nassau, Brasil

E-mail: pedrojordão2@gmail.com

Fernando de Oliveira Martorelli

ORCID: https://orcid.org/0000-0001-7985-4639

Centro Universitário Maurício de Nassau, Brasil

E-mail: fernando.martorelli@hotmail.com

Pedro Paulo Albuquerque Cavalcanti de Albuquerque

ORCID: https://orcid.org/0000-0002-3787-8648

Centro Universitário Maurício de Nassau, Brasil E-mail: pedroalbuquerque2@gmail.com

Larissa Sousa Rangel

ORCID: https://orcid.org/0000-0003-0607-488X

Centro Universitário Maurício de Nassau, Brasil E-mail: larissasouzarangel@ hotmail.com

\begin{abstract}
Resumo
Introdução: Em feridas cirúrgicas, uma das técnicas para auxiliar a cicatrização é a sutura. Após extrações dentarias, o sítio cirúrgico resultante é, na maioria das vezes, cicatrizado por segunda intenção, assim se torna de interesse do cirurgião dentista, reter o coagulo sanguíneo no interior da ferida, assim como manter corpos estranhos fora da própria. Objetivos: O seguinte artigo tem como objetivo demonstrar a técnica de confecção da sutura de paz. Metodologia: A sutura de paz foi aqui descrita em um manequim cirúrgico e é uma modificação do ponto em 8 descrito por Hupp. Para melhor resultados, deve-se usar fios não absorvíveis de nylon monofilamentado. No início, é escolhido um dos lados do sítio cirúrgico, Nesse caso, o vestibular na parte mesial. A primeira perfuração é feita pela agulha nesse ponto de eleição, seguida por uma no lado e parte opostos, nesse caso, distal do lado lingual. Em seguida é perfurada a parte mesial do lado lingual, onde o fio é puxado formado uma alça de aproximadamente $5 \mathrm{~mm}$. A última perfuração é feita na parte distal do lado vestibular e com isso, a agulha é passada por baixo da alça e puxada para a confecção do nó. Após a confecção do nó, 2 formatos de X intercalados verticalmente aparecem no alvéolo. Resultados e conclusão: A sutura de paz propõe uma maior segurança na permanência do coagulo no alvéolo e promove maior proteção contra corpos estranhos. Esse artigo, busca ensinar a usar e promover mais pesquisas para seu melhor entendimento.
\end{abstract}

Palavras-chave: Cirurgia bucal; Suturas; Odontologia.

\begin{abstract}
Introduction: In a surgical woundone of the techniques used to help the healing process is the suture. After dental extractions, the resulting surgical site is, in most cases, healed via secondary healing, making the oral surgeon's best interest to keep the blood clot fixed in the wound and foreign bodies out of it. Objectives: This study has the objective of demonstrating the Paz's suture technique. Methodology: The Paz's suture technique is a modification of the figure of eight technique proposed by Hupp, for better results; use the unabsorvable monofilamented nylon sutures. To start, choose a point in either side, in this case, mesial of the vestibular side. The first puncture is made in this elected point and the second one follows in distal part of the lingual side. The third puncture is made in the mesial part of the lingual side where the suture is pulled and a $5 \mathrm{~mm}$ strap is formed. The final puncture is made in the distal part of the vestibular side where and the needle is past underneath the strap and pulled to form the knot. As a result, two X figures are formed vertically to one another. Results and conclusion: Paz's suture propose better security of the blood clot and an extra barrier against foreign bodies and with this article, we seek to promote researches about Paz's suture to better understand it.
\end{abstract}

Keywords: Surgery, oral; Sutures; Dentistry.

\section{Resumen}

Introducción: En una herida quirúrgica, una das técnicas usadas para ayudar no reparo tejidual es la sutura. Después de una extration dentaria, lo sitio quirúrgica es cicatrizado por secunda intención e con esto, é de interese del dentista mantener lo coagulo en la herida e mientras mantiene cuerpos extraños fuera dela. Objetivos: Lo siguiente artigo tiene 
como objetivo demonstrar e instruir como hacer la técnica de sutura de paz. La sutura de paz es una modificación de la Técnica de sutura en ocho demostrada por Hupp, para mejor resultados use sutura non absorbible de nylon monfilamentado. Para comenzar, elegimos un ponto de início en uno dos lados, en este caso, la parte mesial del lado vestibular. La primera perforación es hecha e en seguida, la segunda en la parte distal del lado lingual. La tercera perforación es hecha en la parte mesial del lado lingual, formando una alza, de aproximadamente $5 \mathrm{~mm}$. La ultima perforación es hecha en la parte distal del lado vestibular e con esto, la aguja es pasada por bajo de la alza e pujada para la confección de lo nulo. Con el nulo hecho, dos formatos de X ira aparecer aliñados verticalmente en lo sitio quirúrgico. Resultados e conclusión: La sutura de paz propone una barrera extra contra cuerpos extraños e una mejor seguranza para lo coagulo sanguíneo. Este artigo busca ensinar el uso e promover pesquisas sobre la sutura de paz para ser mejor comprensíon.

Palabras clave: Cirurgía bucal; Suturas; Odontología.

\section{Introdução}

A sutura é uma técnica de síntese tecidual usada como mecanismo auxiliar do corpo na contenção do coágulo e consequente reparação. Na Odontologia, a sutura é importante para a limitação do acesso e permanência de corpos estranhos no interior do sítio cirúrgico. Além disso, corrobora para o fechamento das áreas com pouco apoio favorecendo uma hemostasia e apreensão do coágulo sanguíneo no interior do alvéolo. (Dragovic et al.,2020; Koshak, 2017; Milloro, Waite, Larsen \& Ghali, 2016; Selvi et al., 2016; Torres-Lagares et al., 2012)

Os protocolos cirúrgicos de remoção dentária ou exodontias tendem a traumatizar o tecido e podem deixar uma cicatriz. Cicatrizações abertas com exposição do tecido ósseo são classificadas como cicatrização por segunda intenção (Hupp, Ellis \& Tucker, 2015; Johal, \& Kreder, 2018; Júnior, Gaujac \& Trento, 2009; Milloro et al., 2016). Tal cicatrização acontece de forma diferente e exige um bom controle da hemostasia e a ausência de corpos estranhos no interior da ferida para seu correto controle e progresso. Suturas simples e como o ponto em 8 podem ser utilizadas em tais casos para melhor controle dos sítios cirúrgicos. (Gay-Escoda, Gómez-Santos, Sánchez-Torres \& Herráez-Vilas, 2015; Huppet al., 2015)

$\mathrm{Na}$ confecção das suturas, o cirurgião dentista tem várias opções na escolha do material de composição do fio, sendo basicamente subdividida entre fios absorvíveis e fios não absorvíveis (Brandt \& Jenkins, 2012; Burkhardt \& Lang, 2015; Dragovic et al., 2020; Pelz, Todtmann, \& Otten, 2015; Villa et al., 2015). Os principais fios não absorvíveis são os de nylon e seda, sendo o nylon mais comumente encontrado na forma monofilamentada, isto é, uma fibra continua da agulha ao fim e a seda sendo multifilamentada, com várias fibras de seda dando forma ao fio. Os fios absorvíveis mais comumente comercializados e indicados para a Odontologia são o de poligalactina 910 (vicryl@) e catgut (Gazivoda, Pelemiš \& Vujašković, 2015; Jardim et al., 2013).

Os fios de nylon e seda, são mais usados entre os calibres 3-0 e 5-0 (Batista et al., 2020; Nadal et al., 2015; Santo, Diniz, Mattos, Filho \& Sá, 2019), desde a microcirurgia periodontal até a cirurgia bucomaxilofacial. O material nylon monofilamentado tem uma grande característica desejável, que é a menor reação inflamatória do organismo. Devido a ser composto de apenas uma fibra continua, não confere espaço a restos de alimento e detritos para ficarem no seu exterior e serem potenciais fatores inflamatórios no local (Asher, Chacartchi, Tandlich, Shapira \& Polak, 2019; Brandt \& Jenkins, 2012; Burkhardt \& Lang, 2015).

Os fios reabsorvíeis CatGut e Vicryl® são consideravelmente mais caros que os de seda e nylon, mas possuem a vantagem de não necessitarem de remoção posterior ao ato cirúrgico. Dito isso, é intrínseco aos seus mecanismos de ação a inflamação do sítio cirúrgico para a absorção (Crosetti, Caracciolo, Arrigoni, Delmastro, \& Succo, 2019; Jardim et al., 2013). Por conta disso, para sítios com alta expectativa de inflamação ou muito dependentes da resposta do corpo para a cicatrização, seu uso é de questionável valia.

A sutura aqui descrita, se compõe de 2 formatos de $\mathrm{X}$ intercalados verticalmente e apenas um nó feito. É confeccionado a partir de 4 perfurações no tecido e uma alça posicionada na região lingual ou mesial. Devido a sua maior área 
de contato, ela oferece barreira extra contra corpos estranhos que possam entrar no alvéolo e também uma proteção contra a evasão do coagulo cicatrizador.

O presente estudo tem como objetivo apresentar e descrever a técnica do "Ponto de paz", assim como estimular pesquisas sobre a mesma, levando a conclusões baseadas no método científico e aprimoramentos da técnica e seu uso.

\section{Metodologia}

O artigo tem como descreve uma modificação especial da técnica da sutura em forma de oito, descrita por Hupp no livro cirurgia e oral e maxilofacial contemporânea.

A demonstração a seguir foi feita logo após a exodontia de um molar inferior em um manequim cirúrgico (Orais, Goiânia, Goiás, Brasil), onde foram utilizados: uma porta agulha de MayoHegar (Golgram São Caetano do sul, São Paulo, Brasil) para manusear a agulha, uma tesoura Goldman Fox (Golgram São Caetano do sul, São Paulo, Brasil) para cortar o fio, uma pinça Addison (Golgram São Caetano do sul, São Paulo, Brasil) para manipular a agulha durante a confecção do ponto. O fio de nylon monofilamentado 4.0 (Technofio, Goiânia, Goiás, Brasil) foi escolhido, por apresentar um menor potencial de reação inflamatória para o tecido. A partir de um alvéolo de um dente recém extraído, a perfuração de um dos lados (vestibular o lingual) é escolhida na porção mesial ou distal de tal alvéolo (Figura 1).

Figura 1. A: Instrumentos utilizados. B: Exodontia. C: Alvéolo cirúrgico.
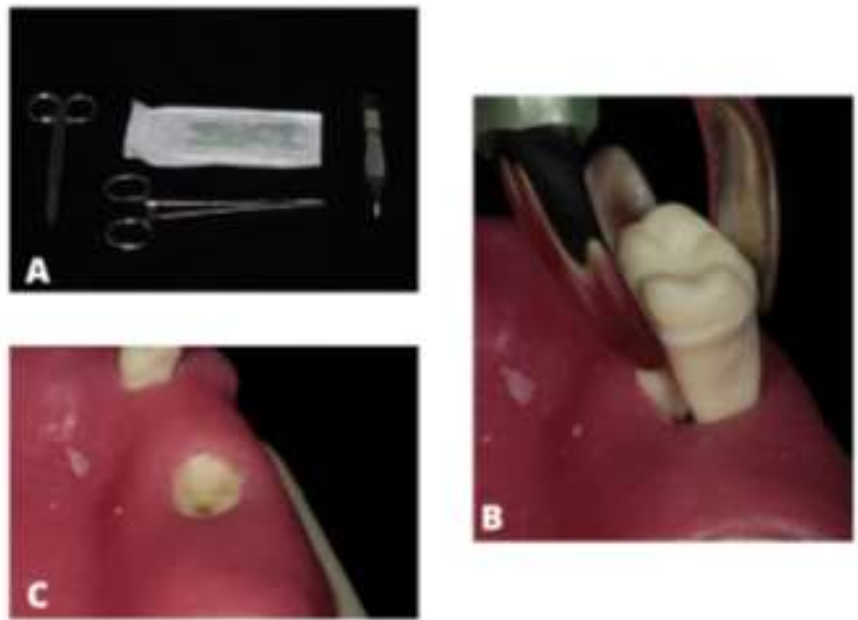

Fonte: Autores.

Para a demonstração foi escolhido o lado vestibular e a porção distal. Após a perfuração do ponto de eleição, a agulha é direcionada a porção mesial do lado lingual, da face interna a externa do sítio cirúrgico. Após isso, ainda no lado lingual, a porção mesial é perfurada da face externa para a interna do alvéolo e o fio é puxado até uma pequena alça de 4 a 6 milímetros de comprimento seja feita (Figura 2). Após isso, uma $4^{\circ}$ e ultima perfuração é feita no alvéolo, no lado lingual e face distal, formando um $\mathrm{X}$ na luz do sítio cirúrgico. 
Figura 2. A: Perfuração distovestibular. B e C: Perfuração mesiolingual. D: Perfuração distolingual.
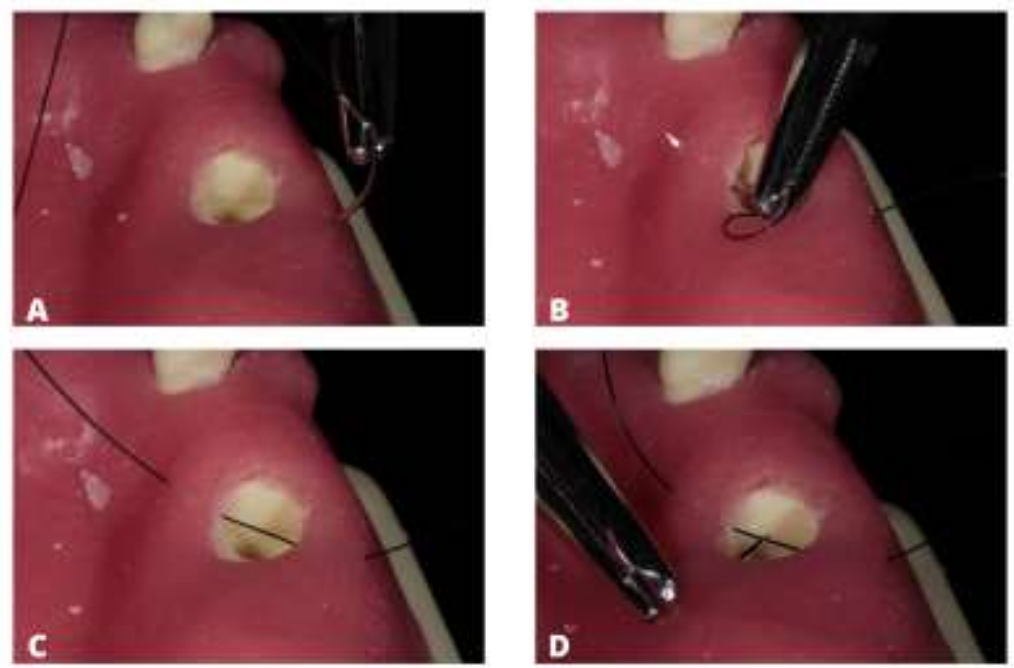

Fonte: Autores.

Feito isso, a agulha é passada por baixo da alça anteriormente feita e o fio puxado até a área onde será confeccionado o nó, o ajustando até a formação de um segundo X sobreposto à primeira foto (Figura 3).

Figura 3. A: Perfuração mesiovestibular. B: Alça e X inicial. C: Agulha sendo passada por baixo da alça. D: Formatos de X sobrepostos verticalmente.
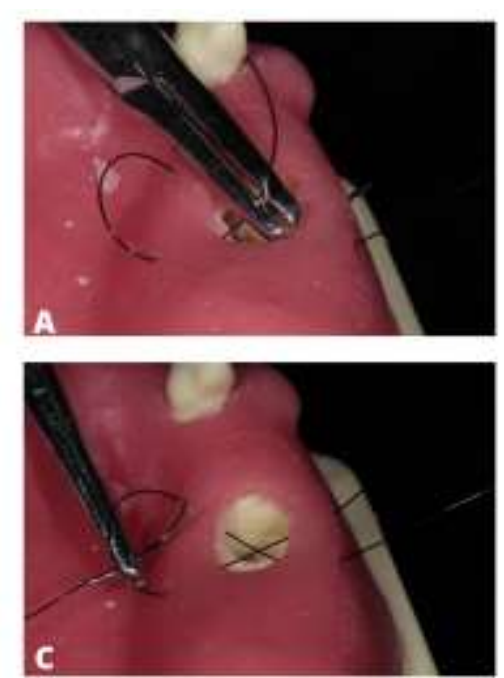
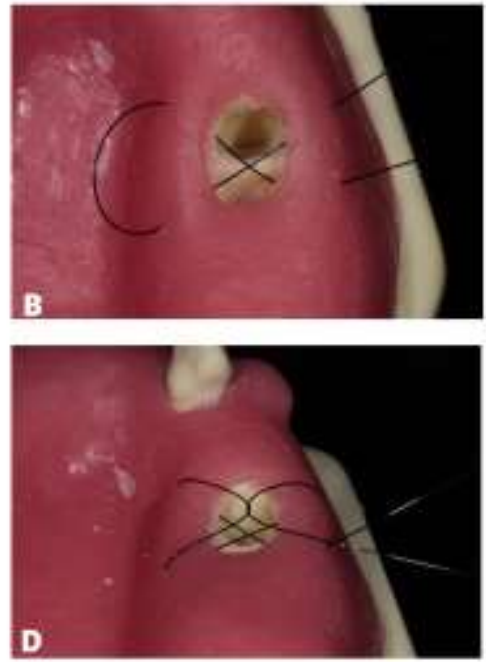

Fonte: Autores.

Após o nó ser feito, o fio de nylon é cortado sem deixar grandes sobras e os 2 formatos de X ficarão sobrepostos e firmes, proporcionando melhor fechamento do sítio com apenas um ponto (Figura 4). 
Figura 4. A: Confecção do nó. B: Produto final.
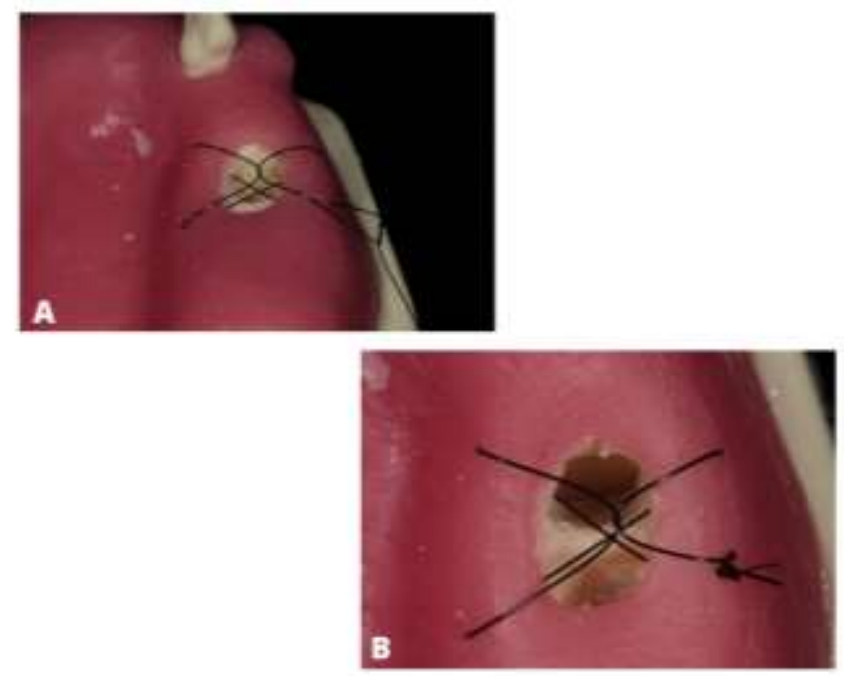

Fonte: Autores.

\section{Resultados e Discussão}

O ponto em 8 duplo, denominado aqui como "Ponto de paz" pelos autores é uma técnica nova que tem como principal vantagem aumentar o fechamento de alvéolos com ponto único, promover mais estabilidade em suturas de feridas fechadas por primeira intenção e ajudar na cicatrização de sítios de doação de tecido conjuntivo. O Ponto de Paz deve ser feito com fios de nylon não absorvíveis monofilamentados, onde o uso de fio de seda deve ser evitado, pelo potencial inflamatório, com suas múltiplas fibras que facilitam a permanência de corpos estranhos e micro-organismos na região suturada.

\section{Considerações Finais}

A ponto de paz requer estudos clínicos para comprovar sua superioridade e viabilidade. Essa demonstração almeja divulgar sua existência e incentivar estudos clínicos usando a técnica para testar sua eficácia.

\section{Agradecimentos}

Os autores agradecem a coordenação do curso de odontologia da Uninassau Campus Boa viagem e ao gestor de educação do grupo ser educacional por disponibilizar o espaço estudo e registro das figuras utilizadas.

\section{Referências}

Asher, R., Chacartchi, T., Tandlich, M., Shapira, L., \& Polak, D. (2019). Microbial accumulation on different suture materials following oral surgery: a randomized controlled study. Clinical oral investigations, 23(2), 559-565.

Batista, T. R. D. M., Pimentel, A. C. S., Silva, F. N. D., Medeiros Neto, M. H. D., \& Tormes, A. K. D. M. (2020). Odontectomia parcial intencional: relato de caso clínico. Rev. cir. traumatol. buco-maxilo-facial, 39-43.

Brandt, M. T., \& Jenkins, W. S. (2012). Suturing principles for the dentoalveolar surgeon. Dental Clinics of North America, 56(1), $281-303$.

Burkhardt, R., \& Lang, N. P. (2015). Influence of suturing on wound healing. Periodontology 2000, 68(1), $270-281$.

Crosetti, E., Caracciolo, A., Arrigoni, G., Delmastro, E., \& Succo, G. (2019). Barbed suture in oral cavity reconstruction: preliminary results. Acta Otorhinolaryngologica Italica, 39(5), 308.

Dragovic, M., Pejovic, M., Stepic, J., Colic, S., Dozic, B., Dragovic, S. \&Milicic, B. (2020). Comparison of four different suture materials in respect to oral wound healing, microbial colonization, tissue reaction and clinical features-randomized clinical study. Clinical oral investigations, $24(4), 1527-1541$. 
Research, Society and Development, v. 10, n. 11, e239101119573, 2021

(CC BY 4.0) | ISSN 2525-3409 | DOI: http://dx.doi.org/10.33448/rsd-v10i11.19573

Dos Santos, M. D. N., do Santo, F., D., Mattos, J. V., Veloso, J. M. D. S. C., \& de Sá, R. C. (2019). Exodontia de mesiodens em palato: relato de caso. Archivesofhealthinvestigation, 8(6).

Gay-Escoda, C., Gómez-Santos, L., Sánchez-Torres, A., \& Herráez-Vilas, J. M. (2015). Effect of the suture technique on postoperative pain, swelling and trismus after removal of lower third molars: A randomized clinical trial. Medicina oral, patologia oral y cirugia bucal, $20(3)$, e372.

Gazivoda, D., Pelemiš, D., \& Vujašković, G. (2015). A clinical study on the influence of suturing material on oral wound healing. Vojnosanitetski pregled, 72(9), 765-769.

Hupp, J. R., Ellis, E. T., \& MR, C. (2015). Cirurgia oral e maxilofacial contemporânea (6a ed.), Elsevier.

Jardim, E. C. G., Paredes, A., Gomes, A. C., Pereira, C. C. S., \& Okamoto, R. (2013). Filho OM. Comparative study of the healing process when using Vicryl®, Vicryl Rapid®, Vicryl Plus®, and Monocryl® sutures in the rat dermal tissue. Oral Maxillofacial Surgery, 17, 293-298.

Johal, H., \& Kreder, H. (2018). Cochrane in CORR®: Negative Pressure Wound Therapy for Skin Grafts and Surgical Wounds Healing by Primary Intention. Clinical orthopaedics and related research, 476(3), 463.

Júnior, L. R. M., Gaujac, C., \& Trento, C. L. (2009). <b> Influência das Alterações Locais Sobre o Processo de Reparo Alveolar. Saúde e Pesquisa, 2(3).

Koshak, H. H. (2017). Dental suturing materials and techniques. Global Journal of Otolaryngology, 12(2), 1-11.

Milloro, M., Waite, P. D., Larsen, P. E. \& Ghali, G. E. (2016) Principios de cirurgia e traumatologia bucomaxilofacial de Peterson (3a ed.), Santos editora.

Nadal, L., Pilatti, A. F., Schwade, F. M. S., Poletto, A. C., Lopes, L. C., \& Fosquiera, E. C. (2015). Exodontia simultânea de terceiros e quartos molares inferiores e superiores: relato de caso clínico. Revista uningá reviewr, 24(1).

Pelz, K., Todtmann, N., \& Otten, J. E. (2015). Comparison of antibacterial-coated and non-coated suture material in intraoral surgery by isolation of adherent bacteria. Annals of Agricultural and Environmental Medicine, 22(3).

Selvi, F., Cakarer, S., Can, T., Topcu, S. İ. K., Palancioglu, A., Keskin, B., \& Keskin, C. (2016). Effects of different suture materials on tissue healing. Journal of Istanbul University Faculty of Dentistry, 50(1), 35.

Torres-Lagares, D., Barranco-Piedra, S., Rodríguez-Caballero, A., Serrera-Figallo, M. A., \& Segura-Egea, J. J., \& Gutiérrez-Pérez, J. L. (2012). Suture needles in Oral Surgery: Alterations depending on the type and number of sutures. Medicina oral, patologia oral y cirugia bucal, 17(1), e129.

Villa, O., Lyngstadaas, S. P., Monjo, M., Satué, M., Rønold, H. J., Petzold, C., \& Wohlfahrt, J. C. (2015). Suture materials affect peri-implant bone healing and implant osseointegration. Journal of oral science, 57(3), 219-227. 